\title{
Prevalence of abnormal Alzheimer's disease biomarkers in patients with subjective cognitive decline: cross-sectional comparison of three European memory clinic samples
}

Steffen Wolfsgruber ${ }^{1,2^{*}}$ (D) José Luis Molinuevo ${ }^{3,4}$, Michael Wagner ${ }^{1,2}$, Charlotte E. Teunissen $^{5}$, Lorena Rami ${ }^{3}$, Nina Coll-Padrós ${ }^{3}$, Femke H. Bouwman ${ }^{6}$, Rosalinde E. R. Slot ${ }^{6}$, Linda M. P. Wesselman ${ }^{6}$, Oliver Peters ${ }^{7}$, Katja Luther ${ }^{7}$, Katharina Buerger ${ }^{8,9}$, Josef Priller ${ }^{10}$, Christoph Laske ${ }^{11,12}$, Stefan Teipel ${ }^{13}$, Annika Spottke ${ }^{2}$, Michael T. Heneka ${ }^{1,2}$, Emrah Düzel $^{14}$, Alexander Drzezga ${ }^{15}$, Jens Wiltfang ${ }^{16,17,21}$, Sietske A. M. Sikkes ${ }^{18,19}$, Wiesje M. van der Flier ${ }^{6,18}$, Frank Jessen ${ }^{2,20}$ and on behalf of the Euro-SCD working group

\begin{abstract}
Introduction: Subjective cognitive decline (SCD) in cognitively unimpaired older individuals has been recognized as an early clinical at-risk state for Alzheimer's disease (AD) dementia and as a target population for future dementia prevention trials. Currently, however, SCD is heterogeneously defined across studies, potentially leading to variations in the prevalence of $A D$ pathology. Here, we compared the prevalence and identified common determinants of abnormal AD biomarkers in SCD across three European memory clinics participating in the European initiative on harmonization of SCD in preclinical AD (Euro-SCD).

Methods: We included three memory clinic SCD samples with available cerebrospinal fluid (CSF) biomaterial (IDIBAPS, Barcelona, Spain, $n=44$; Amsterdam Dementia Cohort (ADC), The Netherlands, $n=50$; DELCODE multicenter study, Germany, $n=42$ ). CSF biomarkers (amyloid beta (Aß)42, tau, and phosphorylated tau (ptau181)) were centrally analyzed in Amsterdam using prespecified cutoffs to define prevalence of pathological biomarker concentrations. We used logistic regression analysis in the combined sample across the three centers to investigate center effects with regard to likelihood of biomarker abnormality while taking potential common predictors (e.g., age, sex, apolipoprotein E (APOE) status, subtle cognitive deficits, depressive symptoms) into account.

Results: The prevalence of abnormal A 422 , but not tau or ptau181, levels was different across centers (64\% DELCODE, $57 \%$ IDIBAPS, 22\% ADC; $p<0.001$ ). Logistic regression analysis revealed that the likelihood of abnormal Aß42 (and also abnormal tau or ptau181) levels was predicted by age and APOE status. For A 342 abnormality, we additionally observed a center effect, indicating between-center heterogeneity not explained by age, APOE, or the other included covariates.

(Continued on next page)
\end{abstract}

\footnotetext{
* Correspondence: Steffen.Wolfsgruber@dzne.de

1 Department of Neurodegeneration and Geriatric Psychiatry, University

Hospital Bonn, Bonn, Germany

${ }^{2}$ German Center for Neurodegenerative Diseases, Sigmund-Freud-Straße 27,

53127 Bonn, Germany

Full list of author information is available at the end of the article
}

(c) The Author(s). 2019 Open Access This article is distributed under the terms of the Creative Commons Attribution 4.0 International License (http://creativecommons.org/licenses/by/4.0/), which permits unrestricted use, distribution, and reproduction in any medium, provided you give appropriate credit to the original author(s) and the source, provide a link to the Creative Commons license, and indicate if changes were made. The Creative Commons Public Domain Dedication waiver (http://creativecommons.org/publicdomain/zero/1.0/) applies to the data made available in this article, unless otherwise stated. 
(Continued from previous page)

Conclusions: While heterogeneous frequency of abnormal A 342 was partly explained by between-sample differences in age range and APOE status, the additional observation of center effects indicates between-center heterogeneity that may be attributed to different recruitment procedures. These findings highlight the need for the development of harmonized recruitment protocols for SCD case definition in multinational studies to achieve similar enrichment rates of preclinical AD.

Keywords: Subjective cognitive decline, Preclinical Alzheimer's disease, CSF biomarkers

\section{Background}

It is widely acknowledged that future prevention and intervention approaches for Alzheimer's disease (AD) will be most effective when applied to individuals in a disease stage prior to mild cognitive impairment (MCI) or prodromal $\mathrm{AD}[1,2]$. As in the latest research framework guidelines proposed for observational and intervention studies [3], AD is defined in vivo by profiling of biomarkers (e.g., those obtained from cerebrospinal fluid (CSF)) grouped into those of amyloid beta deposition (A), pathological tau $(\mathrm{T})$, and those of neurodegeneration $(\mathrm{N})$ in the "AT(N)" system. Preclinical AD is present if patients are cognitively unimpaired and have a biomarker profile of both abnormality in amyloid beta $\left(\mathrm{A}^{+}\right)$ and pathological tau markers $\left(\mathrm{T}^{+}\right)$. Importantly, in this new framework, subjective cognitive decline (SCD) is considered indicative of a stage of transitional cognitive decline, that is between a fully asymptomatic stage and a cognitively impaired (MCI) stage of the disease.

Thus, SCD in cognitively unimpaired older individuals is recognized as a pre-MCI at-risk state of $\mathrm{AD}$ dementia and a target condition for future AD dementia prevention trials. A first set of consensus criteria and research guidelines for operationalization of SCD have been published, but comparability of SCD samples across different research sites is still poor $[4,5]$. Conditions other than AD may cause symptoms of SCD which further promotes heterogeneity. This could be countered by harmonized recruitment protocols including aspects of SCD that enhance the likelihood of underlying $\operatorname{AD}[4,5]$. However, there is currently a lack of such protocols for SCD case definition and assessment in the context of preclinical AD. The design of such a protocol represents a crucial next step before applying SCD in largescale AD prevention trials. To address this issue, the European initiative on harmonization of SCD in preclinical AD (Euro-SCD) aims to develop a harmonized multicenter, multinational case-definition protocol of SCD which should yield comparable rates of preclinical AD (i.e., similar enrichment for AD risk) across memory clinic cohorts. A first step of EURO-SCD, presented in this study, is to retrospectively analyze data from the memory clinic cohorts of the three participating study partners, each recruited with their own
SCD recruitment protocols. Here, our aim was to evaluate the extent of heterogeneity in biomarker abnormality across the three European SCD samples and identifying potential sources for this.

\section{Methods}

Standard protocol approvals, registrations, and patient consent

The study protocol was approved by the Institutional Review Boards of all participating study centers of the Euro-SCD project. All patients provided written informed consent.

\section{Participants}

We analyzed data from three ongoing memory clinic cohorts that recruit SCD participants and collaborate within EURO-SCD: The Amsterdam Dementia Cohort (ADC) $[6,7]$, the cohort of l'Institut d'Investigacions Biomèdiques August Pi i Sunyer Hospital Clinic Barcelona (IDIBAPS) [8], and the German Center for Neurodegenerative Diseases multicenter Longitudinal Cognitive Impairment and Dementia Study (DELCODE) [9]. Each cohort was asked to contribute a target number of 50 or close to 50 SCD patients with available CSF samples. All CSF samples were then centrally analyzed (in Amsterdam) with regard to $\mathrm{AD}$ biomarkers as detailed below. Each cohort is briefly described below, together with its respective SCD recruitment protocol and case definition.

\section{Recruitment procedures and case definition of SCD in each sample DELCODE cohort}

DELCODE is an observational longitudinal memory clinic-based multicenter study carried out by 10 university memory clinics, based within the German Center for Neurodegenerative Diseases (DZNE) research infrastructure. Baseline recruitment started in 2015 and is ongoing. A complete description of DELCODE has been published previously [9]. All SCD patients are referrals, including self-referrals, and all were assessed clinically at the respective memory clinics before entering the study. Assessments included medical history, psychiatric and 
neurological examination, neuropsychological testing, blood laboratory work-up, and routine magnetic resonance imaging (MRI). The German version of the consortium to establish a registry of Alzheimer's disease (CERAD) neuropsychological test battery [10], which includes the Trail-Making Test (TMT) A and B [11], was applied at all memory centers. SCD was defined by the presence of subjectively reported decline in cognitive functioning, experienced as worrisome, and a test performance of better than -1.5 standard deviations (SDs) below age-, education-, and gender-adjusted normal performance [12] on all subtests of the CERAD neuropsychological battery, in line with current SCD research criteria [5]. Additional inclusion criteria were age $\geq 60$ years, fluent German language skills, capacity to provide informed consent, and the presence of a study partner. Main exclusion criteria were conditions which clearly interfere with participation in the study or the study procedures, for example significant sensory impairment, current major depressive episode or other major psychiatric disorders either at baseline or in the past, and chronic use of psychoactive compounds with sedative or anticholinergic effects (see [9] for a full list of inclusion/ exclusion criteria).

\section{Amsterdam Dementia Cohort (ADC)}

The ADC is a cohort consisting of consecutive patients visiting the Alzheimer Center of the VU University Medical Center (VUmc) in Amsterdam, the Netherlands. It has been described in detail previously [6, 7]. All SCD patients underwent a standardized dementia screening, including physical and neurologic examination as well as laboratory tests and brain MRI. Cognitive assessment included the Mini-Mental State Examination (MMSE) and an extensive neuropsychological test battery. Diagnoses were made in a multidisciplinary case conference. Patients were defined as SCD when they presented with cognitive complaints, but cognitive and laboratory investigations were normal and criteria for $\mathrm{MCI}$, dementia, or any other neurologic or psychiatric disorders known to cause cognitive complaints were not met. Petersen's criteria were used for $\mathrm{MCI}$ [13], where the presence of MCI-like objective cognitive impairment was determined by clinical judgment of the complete neuropsychological information rather than applying a specific algorithm or impairment cutoff.

\section{IDIBAPS Barcelona cohort}

The biomarker cohort of the IDIBAPS Hospital Clinic Barcelona recruited patients with SCD, MCI, and AD dementia, as well as cognitively unimpaired participants without cognitive complaints. It has been described in detail previously [8].
Subjects with any neurological diagnosis, serious, or unstable medical condition, or with a diagnosis of major psychiatric disorder including schizophrenia and major depressive, severe somatic disease, or substance abuse were excluded in all the groups. The clinical SCD group comprises subjects who consulted the IDIBAPS Hospital Clinic memory clinic for experience of subjective cognitive decline. They presented normal scores on two screening tests, namely MMSE and the Memory Alteration Test (M@T) [14], and on all subtests of a neuropsychological battery tapping cognitive domains of memory, language, praxis, visuo-perceptive and/or visuospatial ability, and executive functions. Similar to DELCODE, test performance of better than -1.5 SD below the mean of healthy controls, matched for age and education, in all subtests of the applied neuropsychological battery was required for a study diagnosis of SCD.

\section{CSF measures \\ CSF sampling and analytic procedures}

CSF was obtained via lumbar puncture using a 25-gauge needle and collected in 10-ml polypropylene tubes (Sarstedt, Nümbrecht, Germany) in agreement with international consensus protocols [15]. Within $2 \mathrm{~h}$, CSF samples were centrifuged at $4{ }^{\circ} \mathrm{C}$ for $10 \mathrm{~min}$ at $1800 \mathrm{~g}$. CSF supernatant was transferred to 0.5 - $\mathrm{ml}$ polypropylene tubes and stored at $-20^{\circ} \mathrm{C}$ until further analysis (within 2 months) for the Amsterdam samples. Samples collected in the IDIBAPS and DELCODE cohort were stored at $-80^{\circ} \mathrm{C}$ until transfer to Amsterdam for central analysis. Commercially available enzyme-linked immunosorbent assays (ELISAs) (Innotest $\beta$-amyloid(142), InnotestTAU-Ag, and InnotestPhosphotau(181P); Fujirebio, Ghent, Belgium) were applied to measure baseline amyloid beta $(\mathrm{A} \beta) 42$, total tau (t-tau), and tau phosphorylated as position 181 (ptau181) as previously described elsewhere $[16,17]$. Clinical diagnosis was unknown to the team performing the CSF analyses.

\section{Definition of $A D$ biomarker abnormality}

For definition of $\mathrm{AD}$ biomarker abnormality, we applied previously published cutoff values: abnormal CSF-A $\beta 42$ was defined as values $<813 \mathrm{pg} / \mathrm{ml}$ [17]; abnormal CSF t-tau was defined as values $>375 \mathrm{pg} / \mathrm{ml}$; and CSF-ptau181 was defined as values $>53 \mathrm{pg} / \mathrm{ml}$ [18]. Besides abnormality in individual markers, we also report results for a CSF-based operationalization of preclinical $\mathrm{AD}$ according to the most recent National Institute on Aging and the Alzheimer's Association (NIA-AA) criteria [3], that is defined by the presence of both abnormal CSF-A 42 and CSF-ptau181.

\section{Clinical and neuropsychological assessment}

Clinical and neuropsychological assessment was carried out in each center following center-specific standardized 
diagnostic procedures that have been described in other publications (ADC [6, 7], IDIBAPS [19], and DELCODE [9]). Here, we only report the assessments relevant to the present study. For the clinical and neuropsychological data to be used as predictors of CSF abnormality across samples, we applied several rescaling procedures as described below.

\section{Assessment of neuropsychological test performance}

All centers applied established neuropsychological tests mainly covering three cognitive domains. Verbal memory was assessed with the German Version of the CERAD wordlist [10] in DELCODE, the Dutch version of the Rey Verbal Learning Test (RVLT) [20] in ADC, and the Spanish version of the Free and Cued Selective Reminding Test (FCSRT) [21] in the IDIBAPS sample. Executive functions and speed were measured by the TMT-A and TMT-B [11] in all three samples. Language abilities were measured by tests of semantic verbal fluency (animals) in all three samples [22]. The DELCODE and IDIBAPS sample also applied the 15-item version of the Boston Naming Test (BNT) [22], while a second, verbal fluency measure (letters) was available in the ADC sample [22].

We used center-specific normative data to derive age-, sex-, and education-adjusted $z$-scores for each sample. For each participant we then aggregated the $z$-score information of the available tests in the three cognitive domains into a single, dichotomized variable with the categories "evidence of subtle cognitive decline" vs. "no evidence of subtle cognitive decline". We derived this variable by adapting the method proposed by Edmonds and colleagues [23]: "evidence of subtle cognitive decline" was defined by performance of more than $1 \mathrm{SD}$ below the normative mean (i.e., a $z$-score $<-1$ ) on at least two out of six preselected neuropsychological measures (two of each of the three different cognitive domains described above). For verbal memory we used the word list delayed recall and recognition scores from the CERAD in DELCODE and from the RVLT in the ADC sample, respectively. The best equivalent to this in the IDIBAPS sample was the FCSRT free and total recall score [24]. In the language abilities domain, we utilized the animal fluency and BNT score in IDIBAPS and DELCODE, and animal + letter fluency score in the ADC sample.

\section{Depressive symptomatology and instrumental activities of daily living performance}

Depressive symptomatology was measured with the 15-item version of the Geriatric Depression Scale (GDS) [25] in DELCODE and ADC where a cutoff $>5$ indicates depressive symptomatology. In IDIBAPS, depressive symptoms were measured using the Hospital Anxiety and Depression Scale (HADS) [26], where a cutoff $>7$ indicates depressive symptomatology.

Instrumental activities of daily living (IADL) were assessed with the Functional Activities Questionnaire (FAQ) [27] in IDIBAPS and DELCODE and with the Disability Assessment for Dementia (DAD) scale in the ADC sample [28]. Due to the limited range and variance in SCD patients on these two respective measures, we derived a dichotomized variable with the following categories: "no IADL deficits" (fully unimpaired) vs. "subtle IADL deficits" (mildly imperfect performance, that is a score $\geq 1$ on the FAQ or a score $<100$ on the DAD, respectively).

\section{Statistical analysis}

Statistical analyses were conducted with SPSS version 22. As this is an exploratory rather than a confirmatory analysis, we report unadjusted $p$ values.

Our main analysis focused on evaluating heterogeneity of biomarker abnormality across the three subsamples and identifying potential sources for this. In that regard, we conducted four separate stepwise logistic regression analyses, i.e., one analysis each for abnormal CSF-A $\beta 42$, CSF-tau, CSF-ptau181, and the aforementioned NIA-AA preclinical $\mathrm{AD}$ definition (i.e., presence of abnormal CSF-A 442 together with abnormal CSF-ptau181; [3]) as the dependent variable, respectively.

We included the following covariates/predictors in a forward selection procedure $(p$ value for inclusion $\leq 0.05)$ in step one: age, sex, years of education, evidence of subtle cognitive decline, and apolipoprotein E (APOE) genotype. For insignificant predictors we will report the chi-squared values of the score test with the corresponding $p$ values. The score test, or Lagrange multiplier test, is used in forward selection procedures to test for improvement of model fit if variables are successively added to a prediction model. If there was any cross-center heterogeneity in biomarker abnormality not explained by the predictors of step one, then a categorical predictor of "study-center" should significantly improve model fit by increasing explained variance beyond that of the covariate model. Therefore, we entered study center as an additional predictor in a second step. This main analysis was based on a sample of $n=132$ cases (four cases with missing APOE).

In a supplementary analysis, we repeated the aforementioned regression models with additional inclusion of depressive symptomatology and subtle IADL deficits as predictors in step one. This was based on a restricted sample with $n=92$ cases $(n=44$ cases not included due to missing data on IADL performance $(n=39)$, depressive symptoms $(n=10)$, and/or APOE $(n=4)$; some subjects had missing values on more than one of these variables). 
Cases with missing data did not differ regarding age, education, MMSE score, sex or (for those with missing on depression or IADL only) APOE status, suggesting that the assumption of a missing (completely) at random data pattern was not violated, thus allowing for either multiple imputation of the missing depression and IADL scale values or a complete case analysis. For the sake of simplicity, we report on the latter, as a sensitivity analysis using multiple imputation did not change the results.

\section{Results}

\section{Descriptive statistics}

Descriptive statistics of the whole sample and each subsample regarding demographical, clinical, neuropsychological, and biomarker data are given in Table 1 .

The mean age substantially varied between the centers, with the ADC participants (mean \pm SD, $62.9 \pm 6.1$ years) being on average about 3.5 years younger than those of IDIBAPS (66.2 \pm 7.2 years) and about 8 years younger than those of DELCODE $(71.2 \pm 4.8)$. We further observed differences between the samples regarding sex (with IDIBAPS containing a substantially higher number of women), education, frequency of subclinical depressive symptomatology, and neuropsychological variables. Frequency of positive APOE4 status (overall 35.6\%) did not differ between the three samples. As expected, neuropsychological performance was in the range of cognitive normality, with average norm-adjusted $z$-scores between +0.5 and $-0.5 \mathrm{SD}$, in all samples. However, there was still variation within this range as indicated by significant between-center differences in the number of participants meeting the neuropsychological criteria [23] used to define "evidence of subtle cognitive decline" (9.1\% in IDIBAPS, $14.3 \%$ in DELCODE, and $42 \%$ in ADC, $p=0.001$ ).

Regarding the CSF biomarkers, distribution for each center in the form of boxplots is shown in Fig. 1. We observed (pooled) frequencies of abnormal CSF-A 342 in $46.3 \%$, abnormal CSF-tau in $25.7 \%$, and abnormal CSF-ptau181 in $40.4 \%$ of all SCD patients. CSF-defined preclinical AD (both abnormal CSF-A $\beta 42$ and CSF-ptau181) was observed in $18.4 \%$.

\section{Between-center heterogeneity in AD biomarker abnormality}

On a descriptive level (i.e., not accounting for covariates) only the CSF-A $\beta 42$ levels were significantly different across the centers, both in terms of continuous values and frequency of abnormal CSF-A $\beta 42$ according to the applied cutoff $(<813 \mathrm{pg} / \mathrm{ml} ; 64 \%$ DELCODE, 57\% IDIBAPS, 22\% ADC; $p<0.001$, see Table 1 and Fig. 1 ).

Results of the logistic regression analyses for prediction of each abnormal CSF biomarker are presented in Table 2. Higher age and a positive APOE status (i.e., at least one E4 allele) were associated with a higher likelihood of CSF-A $\beta 42$ abnormality. Sex (score test $\chi^{2}$ (df $=1)=0.027, p=0.871$ ), years of education (score test $X^{2}(\mathrm{df}$ $=1)=0.334, p=0.563)$, and evidence of subtle cognitive decline (score test $X_{(\mathrm{df}=1)}^{2}=0.044, p=0.833$ ) did not show a significant association.

After adjusting for age and APOE status in step 1, we observed a significant center effect, such that both IDIBAPS (odds ratio (OR) 5.78, 95\% confidence interval (CI) 2.14-15.6) and DELCODE (OR 6.54, 95\% CI 2.1420.0) had similarly increased risk of abnormal A 442 values compared with ADC.

Higher age and positive APOE status were also associated with higher likelihood of having abnormal CSF-tau as well as higher likelihood of fulfilling the CSF-based preclinical AD definition. For CSF-ptau181 abnormality, only APOE4 was a significant covariate. Other covariate effects were not observed for these markers (for tau: sex, score test $X_{2}^{2}(\mathrm{df}=1)=0.08, p=0.777$; years of education, score test $\chi_{(\mathrm{df}=1)}^{2}=0.014, p=0.906$; evidence of subtle cognitive decline, score test $X_{(\mathrm{df}=1)}^{2}=0.254, p=0.614$; for ptau181: age, score test $\chi_{(\mathrm{df}=1)}^{2}=0.474, p=0.491$; sex, score test $\chi_{(\mathrm{df}=1)}^{2}=0.074, p=0.785$; years of education, score test $\chi_{(\mathrm{df}=1)}^{2}=0.033, p=0.857$; evidence of subtle cognitive decline, score test $\chi_{(\mathrm{df}=1)}^{2}=2.45, p=0.118$; for the CSF-based preclinical AD definition: sex, score test $\chi^{2}(\mathrm{df}=1)=0.08, p=0.777$; years of education, score test $X^{2}(\mathrm{df}=1)=0.014, p=0.906$; evidence of subtle cognitive decline, score test $\left.\chi_{(\mathrm{df}=1)}^{2}=0.254, p=0.614\right)$.

There was also no center effect for these markers (see Table 2).

The supplementary analysis with IADL and depressive symptomatology as additional predictors showed similar results with regard to the effects reported above. Subtle IADL deficits and depressive symptomatology were not associated with likelihood of abnormality in any biomarker.

\section{Discussion}

The aim of the present study was to compare the prevalence of CSF biomarker abnormality across three different memory clinic samples that collaborate within the Euro-SCD project and to identify predictors of abnormal CSF from a set of variables available in all subsamples, such as age, APOE genotype, depressive symptomatology, and neuropsychological and IADL performance. In addition, we determined, by testing for center effects, whether there was significant between-center heterogeneity with regard to CSF abnormality after adjusting for the aforementioned factors.

Unadjusted for any covariates, we observed significantly different frequencies of biomarker abnormality between the samples only for CSF-A $\beta 42$. DELCODE had the highest prevalence of $\mathrm{A} \beta 42$ abnormality (64\%), followed by IDIBAPS (57\%) and ADC (22\%). Results of 
Table 1 Baseline characteristics of the whole study sample

\begin{tabular}{|c|c|c|c|c|c|c|}
\hline \multirow[t]{2}{*}{ Characteristic } & \multirow{2}{*}{$\begin{array}{l}\text { Whole sample } \\
(n=136)\end{array}$} & \multirow{2}{*}{$\begin{array}{l}\text { DELCODE } \\
(n=42)\end{array}$} & \multirow{2}{*}{$\begin{array}{l}\text { IDIBAPS } \\
(n=44)\end{array}$} & \multirow[t]{2}{*}{$\mathrm{ADC}(n=50)$} & \multicolumn{2}{|c|}{ Between center differences (ANOVA $\left./ x^{2}\right)$} \\
\hline & & & & & $\overline{F / X^{2}(d f=3)}$ & $p$ value \\
\hline Age, years & $66.5 \pm 7.0$ & $71.2 \pm 4.8$ & $66.2 \pm 7.2$ & $62.9 \pm 6.1$ & 21.0 & $<0.001$ \\
\hline Education, years & $12.5 \pm 4.2$ & $14.9 \pm 3.4$ & $10.3 \pm 4.6$ & $12.4 \pm 3.2$ & 15.6 & $<0.001$ \\
\hline Sex, female (\%) & 51.5 & 42.9 & 79.5 & 34.0 & 21.2 & $<0.001$ \\
\hline MMSE total score & $28.4 \pm 1.5$ & $29.0 \pm 1.0$ & $28.0 \pm 1.7$ & $28.1 \pm 1.6$ & 6.8 & 0.002 \\
\hline FAQ score ${ }^{a}$ & - & $0.80 \pm 1.10$ & $1.05 \pm 1.75$ & - & - & - \\
\hline DAD score ${ }^{b}$ & - & - & - & $96.70 \pm 5.70$ & - & - \\
\hline Subtle IADL deficits ${ }^{c}(\%)$ & 45.4 & 46.3 & 47.6 & 42.9 & 0.148 & 0.929 \\
\hline Evidence of depressive symptoms ${ }^{\mathrm{d}}(\%)$ & 8.80 & 0.0 & 17.9 & 10.9 & 7.6 & 0.022 \\
\hline Verbal Delayed Recall, z-score & $-0.04 \pm 1.11$ & $0.33 \pm 1.00$ & $0.11 \pm 0.98$ & $-0.49 \pm 1.17$ & 7.5 & 0.001 \\
\hline Recognition/Cued Recall, z-score & $0.27 \pm 0.90$ & $0.49 \pm 0.57$ & $0.35 \pm 1.01$ & $0.00 \pm 0.96$ & 3.8 & 0.025 \\
\hline Verbal Fluency Animals, z-score & $0.00 \pm 0.91$ & $0.31 \pm 0.96$ & $0.06 \pm 0.73$ & $-0.31 \pm 0.92$ & 5.6 & 0.005 \\
\hline Verbal Fluency Letters ${ }^{b}, z$-score & - & - & - & $0.36 \pm 1.12$ & - & - \\
\hline Boston Naming ${ }^{a}, z$-score & - & $0.63 \pm 0.65$ & $0.30 \pm 0.83$ & - & - & - \\
\hline TMT-A, z-score & $-0.09 \pm 1.04$ & $0.30 \pm 1.20$ & $0.02 \pm 0.97$ & $-0.50 \pm 0.81$ & 7.7 & 0.001 \\
\hline TMT-B, z-score & $-0.15 \pm 1.00$ & $0.36 \pm 1.09$ & $-0.40 \pm 1.01$ & $-0.39 \pm 0.72$ & 9.3 & $<0.001$ \\
\hline Evidence of subtle cognitive decline ${ }^{e}(\%)$ & 22.8 & 14.3 & 9.1 & 42.0 & 16.9 & 0.001 \\
\hline Apolipoprotein E ع4 carriers (\%) & 35.6 & 28.2 & 32.6 & 44.0 & 2.6 & 0.267 \\
\hline $\mathrm{A} \beta 42, \mathrm{pg} / \mathrm{ml}$ & $860.8 \pm 298.1$ & $740.0 \pm 216.8$ & $741.0 \pm 236.2$ & $1067.8 \pm 295.9$ & 7,8 & $<0.001$ \\
\hline $\mathrm{A} \beta 42<813 \mathrm{pg} / \mathrm{ml}(\%)$ & 46.3 & 64.3 & 56.8 & 22.0 & 19.3 & $<0.001$ \\
\hline $\mathrm{t}$-tau, pg/ml & $352.5 \pm 280.8$ & $322.4 \pm 193.4$ & $378.5 \pm 314.6$ & $354.9 \pm 312.6$ & 0.428 & 0.653 \\
\hline t-tau > 470 pg/ml (\%) & 25.7 & 31.0 & 29.5 & 18.0 & 2.5 & 0.287 \\
\hline ptau181, pg/ml & $55.5 \pm 28.7$ & $51.9 \pm 22.4$ & $59.2 \pm 34.2$ & $55.4 \pm 28.4$ & 0.684 & 0.506 \\
\hline ptau181 > 57 pg/ml (\%) & 40.4 & 35.7 & 45.5 & 40.0 & 0.853 & 0.653 \\
\hline Aß42 and ptau181 abnormal (\%) & 16.2 & 21.4 & 20.5 & 8.0 & 3.9 & 0.141 \\
\hline
\end{tabular}

Table shows sample description for the whole study sample and each center's subsample of SCD participants

Values are shown as mean \pm standard deviation unless otherwise indicated

Significant $p$ values are indicated in bold typeface

$Z$-scores for neuropsychological variables represent age-, sex-, and education-adjusted normative values after applying center-specific norms in all study sites (see Methods section for further details)

$A \beta$ amyloid beta, $A D C$ Amsterdam Dementia Cohort, ANOVA analysis of variance, DAD Disability Assessment for Dementia, DELCODE German Center for Neurodegenerative Diseases (DZNE) multicenter Longitudinal Cognitive Impairment and Dementia Study, FAQ Functional Activities Questionnaire, GDS Geriatric Depression Scale, HADS Hospital Anxiety and Depression Scale, IADL instrumental activities of daily living, IDIBAPS I'Institut d'Investigacions Biomèdiques August Pi i Sunyer Hospital Clinic Barcelona, MMSE Mini-Mental State Examination, ptau181 tau phosphorylated at position 181, TMT Trail-Making Test, $t$-tau total tau

a Assessed only in DELCODE and IDIBAPS

${ }^{b}$ Assessed only in ADC

' Subtle IADL deficits defined as mildly imperfect performance, i.e., a score $\geq 1$ on the $F A Q$, or a score $<100$ on the $D A D$, respectively

d Evidence of depressive symptoms defined as a depression score above the cutoff of the applied scale (DELCODE and ADC, GDS >5; IDIBAPS, HADS > 7)

${ }^{e}$ Evidence of subtle cognitive decline operationalized according to criteria proposed by Edmonds and colleagues (see [23] and Methods section): impaired score of $>1$ SD below normative mean (i.e., $z$-score $<-1$ ) on two measures in different cognitive domains

the logistic regression analyses suggest that this apparent heterogeneity in A 442 abnormality between the samples was to some extent explained by age and APOE4 status, two factors for which an association with likelihood of amyloid pathology is already well established (e.g., [29]). Age had a stronger influence on A $\beta 42$ abnormality than APOE4 status (see Table 2 for each predictor's contribution of explained variance in $R^{2}$ ) and the samples differed more strongly in mean age compared with frequency of APOE4 status (see Table 1). With regard to the aim of achieving homogeneous and enriched rates of amyloid positivity across multiple SCD cohorts, our results suggest that these two factors need to be addressed in a harmonized case-definition protocol. This could mean definition of similar age strata or a minimum age cutoff (e.g., $>60$ years) as inclusion criterion while positive APOE status could be used to define subgroups of SCD patients even further enriched for preclinical AD. On the other hand, APOE status can be used as a balancing factor when 

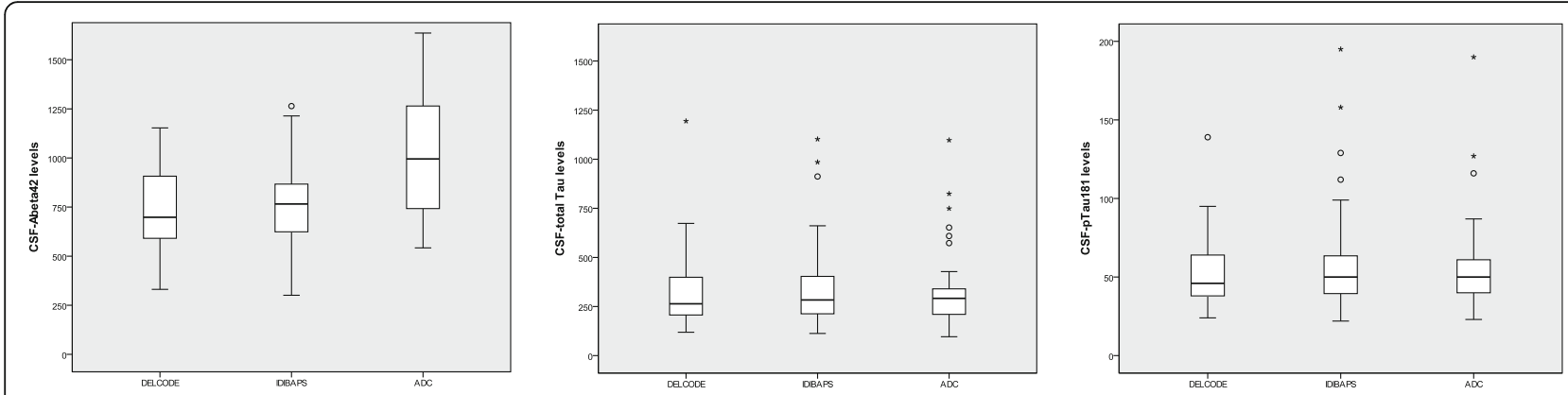

Fig. 1 Boxplots for cerebrospinal fluid (CSF) levels in each sample of the three participating Euro-SCD centers. Values are presented in pg/ml. Points mark individual values lying outside 1.5 times the interquartile range (25-75\% percentile). Asterisks mark extreme values lying outside three times the interquartile range. "The DELCODE and IDIBAPS sample show significantly lower levels compared with the ADC sample ( $p<0.001)$. ADC Amsterdam Dementia Cohort, DELCODE German Center for Neurodegenerative Diseases (DZNE) multicenter Longitudinal Cognitive Impairment and Dementia Study, IDIBAPS I'Institut d'Investigacions Biomèdiques August Pi i Sunyer Hospital Clinic Barcelona

selecting samples across multiple sites since selection of SCD samples with equal rates of APOE4 will increase cross-sample homogeneity with regard to the presence of preclinical AD.

However, even after adjusting for age and APOE status, we did observe significant variance in the likelihood of amyloid positivity between the centers which was of equal magnitude other than the portion of variance explained by age and APOE (see Table 2). The presence of this center-effect after accounting for age and APOE suggests that there are further, unmeasured factors which differed between centers and influenced the likelihood of amyloid pathology. This may include different referral procedures to the memory clinics across the three countries and differences in the center-specific
SCD recruitment protocols, for example the application of different criteria to exclude MCI. Recruitment methods and sources of referral pose a major challenge to standardize (e.g., due to country-specific differences in the healthcare system) and also to quantify in a way that their influence can be assessed and controlled for in future studies.

Moreover, quantitative and qualitative information of the exact nature of the SCD experience may give additional, valuable information with regard to (the differences in) $\mathrm{AD}$ risk, as already suggested by the "SCD plus" criteria $[5,30]$. This could inform recruitment protocols for SCD case definition in research studies. In the present study, we could not analyze this due to lack of data since a common continuous measurement of SCD

Table 2 Results of logistic regression analysis for prediction of abnormal CSF biomarkers across all three centers

\begin{tabular}{|c|c|c|c|c|c|c|c|c|c|c|}
\hline Model/predictors & OR & $(95 \% \mathrm{Cl})$ & $p$ value & $R^{2}$ & $\Delta R^{2}$ & OR & $(95 \% \mathrm{Cl})$ & $p$ value & $R^{2}$ & $\Delta R^{2}$ \\
\hline Model variables & \multicolumn{5}{|c|}{ Abnormal CSF-Aß42 (<813 pg/ml) } & \multicolumn{5}{|c|}{ Abnormal CSF-tau (> 375 pg/ml) } \\
\hline \multicolumn{11}{|l|}{ Step 1: covariates } \\
\hline Age (per 1-year increase) & 1.09 & $1.03-1.15$ & 0.003 & 0.080 & 0.080 & 1.18 & $1.09-1.28$ & $<0.001$ & 0.187 & 0.187 \\
\hline Positive APOE $\varepsilon 4$ status & 2.34 & $1.10-5.00$ & 0.028 & 0.126 & 0.046 & 6.20 & $2371-16.2$ & $<0.001$ & 0.329 & 0.142 \\
\hline Step 2: center (ADC = reference) & & & 0.001 & 0.271 & 0.145 & & & 0.562 & 0.339 & 0.010 \\
\hline DELCODE & 6.54 & $2.14-20.0$ & 0.001 & & & 0.841 & $0.241-2.94$ & 0.786 & & \\
\hline IDIBAPS & 5.78 & $2.14-15.6$ & 0.001 & & & 1.53 & $0.476-4.89$ & 0.477 & & \\
\hline Model variables & \multicolumn{5}{|c|}{ Abnormal CSF-ptau181 (> 53 pg/ml) } & \multicolumn{5}{|c|}{ Abnormal A $\beta 42$ and abnormal ptau181 } \\
\hline \multicolumn{11}{|l|}{ Step 1: covariates } \\
\hline Age (per 1-year increase) & \multicolumn{5}{|c|}{ Not selected } & 1.15 & $1.05-1.26$ & 0.002 & 0.117 & 0.117 \\
\hline Positive APOE ع4 status & 2.66 & $1.28-5.54$ & 0.009 & 0.069 & 0.069 & 5.23 & $1.80-15.2$ & 0.002 & 0.234 & 0.117 \\
\hline Step 2: center (ADC = reference) & & & 0.728 & 0.075 & 0.006 & & & 0.393 & 0.255 & 0.021 \\
\hline DELCODE & 0.983 & $0.401-2.41$ & 0.970 & & & 1.68 & $0.374-7.54$ & 0.499 & & \\
\hline IDIBAPS & 1352 & $0.574-3.19$ & 0.490 & & & 2.60 & $0.643-10.5$ & 0.180 & & \\
\hline
\end{tabular}

$R^{2}$ and $\Delta R^{2}$ give an estimate of explained variance of the full model and each predictor's contribution of explained variance within the model, respectively No values are reported for nonsignificant predictor variables in step 1 of the models, as we used a forward selection process at this step

$A \beta$ amyloid beta, $A D C$ Amsterdam Dementia Cohort, $A P O E$ apolipoprotein $\mathrm{E}$, Cl confidence interval, CSF cerebrospinal fluid, DELCODE German Center for Neurodegenerative Diseases (DZNE), IDIBAPS I'Institut d'Investigacions Biomèdiques August Pi i Sunyer Hospital Clinic Barcelona, OR odds ratio, ptau181 tau phosphorylated at position 181 
was not available and joint analysis of differing scales would require advanced psychometric (i.e., item-response theory) techniques for which the sample size of the present study was not sufficient. The association of specific questionnaire items with biomarkers of $\mathrm{AD}$ in $\mathrm{SCD}$ is investigated in an ongoing multicohort project of the SCD Initiative ("item analysis project" [5]). Furthermore, we will address the relationship of the "SCD plus" features with AD biomarkers in each of the Euro-SCD cohorts in a future joint study. For this study, subjects will be prospectively recruited at each site with a harmonized SCD case definition protocol that also includes common assessment of the SCD plus features.

It is further noteworthy that, besides the observed differences in $A \beta 42$ abnormality, the relative frequency of those with abnormal ptau181 within the group of amyloid positives was relatively equal across samples as can be seen by comparing the frequency of $A \beta 42$ abnormality with the frequency of combined $A \beta 42$ and ptau181 abnormality in Table 1 (DELCODE, 64.3/21.4 $=2.85$; IDIBAPS, $56.8 / 20.5=2.77$; and ADC, 22.0/8.0 $=2.75$ ). Thus, around every third SCD patient with amyloid abnormality also had abnormal ptau181 and would thus fulfill preclinical $A D$ criteria according to the most recent guidelines [3]. At the same time, however, the total number of subjects with ptau181 abnormality in the ADC cohort is relatively high when seen in relation to its overall lower amyloid positivity. This may suggest that this sample also contained, to a higher degree, SCD patients with tau pathology in the absence of a prototypical, amyloid-induced, AD pathological process ("non-AD pathological change" [3]). However, this is speculative, and potential reasons remain elusive to us at the moment. In this regard it is also worth mentioning that a relatively higher frequency of ptau181 abnormality compared with $\mathrm{A} \beta 42$ abnormality has also been reported in an earlier longitudinal SCD study of the ADC cohort [31]. In this study, however, isolated increased tau/ ptau181 was not associated with future cognitive decline, so it might be partly nonspecific.

Of note, the high prevalence of $A \beta 42$ abnormality reported here for the SCD patients from DELCODE and IDIBAPS is higher compared with that reported for SCD patients in earlier reports using these cohorts $[8,9]$. However, this can be explained by the lower (i.e., stricter) cutoffs applied in these aforementioned studies. Of note, the cutoff for the present analysis is based on reference data from the ADC cohort, Gaussian mixture modeling [16], and adjusted for cohort-nonspecific upward drift in Innotest results [17]. Thus, it was the most suited cutoff for our central CSF analysis and, although leading to the aforementioned discrepancies compared with earlier reports for DELCODE and IDIBAPS, does not introduce a bias for the between-center analysis in the present study. Furthermore, it has recently been shown that differences in storage time (DELCODE recruitment started more recently than ADC and IDIBAPS) has no clear effect on CSF A $\beta 42$, tau, and ptau181 values [32]. Nevertheless, we still acknowledge that differences in CSF handling and storage or the shipment of DELCODE/IDIBAPS probes to the central CSF analysis in the ADC center may have influenced our results. However, taken together, we propose that our central CSF analysis is a clear strength of the present study, that is it improved the validity of our outcome data rather than posing a serious source of bias.

Subtle IADL deficits did not predict CSF biomarker abnormality in the present sample. Of note, SCD and MCI patients display (by definition) largely preserved IADL functions. Therefore, the fact that we did not observe an association of subtle IADL deficits with abnormal CSF markers in the present study might be due to limitations in measurement, as the FAQ and DAD scale are not designed to capture very subtle deficits. This is underlined by the finding of ceiling effects (i.e., "fully unimpaired") in both scales. In fact, a recent study, using a more refined IADL measure, demonstrated an increase in IADL impairment across the spectrum from cognitively normal (without subjective cognitive complaints) to $\mathrm{MCI}$, with SCD subject's IADL performance lying in between [33]. This further highlights the importance of a refined characterization of very early functional deficits in this group.

Previous studies have also shown, albeit modest, associations between subtle cognitive performance deficits and $\mathrm{AD}$ biomarkers in cognitively normal samples not recruited through memory clinics (e.g., [34, 35]). While in the present, memory clinic-based study the average neuropsychological performance in each subsample was well above the range of MCI-level impairment, we observed differences regarding the frequency of subjects classified as having evidence of subtle cognitive decline according to the method of Edmonds and colleagues (see Methods section and [23]). While DELCODE (14\%) and IDIBAPS (9\%) had a similar rate of subjects with evidence of subtle cognitive decline, it was considerably higher in ADC with about $40 \%$ of subjects fulfilling the criteria. Of note, this pattern might reflect that operationalization of $\mathrm{MCI}$ as an exclusion criterion in IDIBAPS and DELCODE was very similar (i.e., based on predefined impairment cutoffs). This approach differs from that of ADC where definition of MCI was based on clinical judgment of the complete neuropsychological information rather than applying a specific algorithm or impairment cutoff. While both are valid and commonly used approaches to exclude MCI in the definition of SCD [5], they may still lead to heterogeneity across the resulting samples in terms of neuropsychological 
performance. However, despite this discrepancy, evidence of subtle cognitive decline was not associated with higher likelihood of CSF abnormality. These results are in line with data from a large cohort study that showed equal risk for incident $\mathrm{AD}$ dementia in individuals with unimpaired memory performance but memory concerns compared with those with a study diagnosis of "early MCI" (i.e., conceptually similar to the subtle cognitive decline operationalization used here) [30]. This challenges the usefulness of subtle cognitive deficits as a predictor for $\mathrm{AD}$ risk in pre-MCI samples. However, a possible alternative explanation for the negative finding, and likewise for the observed differences regarding evidence of subtle cognitive decline, might be that our operationalization relied on center-specific normative data (and, to some extent, on nonidentical tests per domain in each center). The resulting $z$-scores thus only represent relative deficit scores compared with each center's specific normative data. As a consequence, their comparability in an absolute manner is not feasible. Limited comparability of different normative samples and the fact that norms might be differently accurate for different age strata preclude this. These factors may have stirred the between-center differences regarding the number of SCD patients meeting the subtle cognitive decline criteria and, likewise, may have precluded the finding of an association between subtle cognitive performance deficits and biomarker abnormality in the present sample.

With regard to homogenous SCD sample selection, close attention should therefore be paid to enabling a homogenous neuropsychological characterization (ideally including comparable norms) to enable a valid, neuropsychological MCI definition as an exclusion criterion across different samples. Application of such a common MCI criterion may further reduce heterogeneity with regard to the prevalence of preclinical AD. However, this needs to be empirically tested in a future multisite study.

\section{Conclusions}

In summary, the results of the present study emphasize the need for harmonized SCD case-definition protocols for future studies on intervention and prevention in this promising target group. Age range, genetic risk factors, and cognitive functional status are important factors to be considered in the development of such protocols aiming to achieve similar enrichment rates of preclinical AD.

\section{Abbreviations}

AD: Alzheimer's disease; ADC: Amsterdam Dementia Cohort; APOE: Apolipoprotein E; Aß: Amyloid beta; BNT: Boston Naming Test; CERAD: Consortium to establish a registry of Alzheimer's disease; Cl: Confidence interval; CSF: Cerebrospinal fluid; DAD: Disability Assessment for Dementia; DELCODE: German Center for Neurodegenerative Diseases multicenter Longitudinal Cognitive Impairment and Dementia Study; EuroSCD: European initiative on harmonization of subjective cognitive decline in preclinical Alzheimer's disease; FAQ: Functional Activities Questionnaire; FCSRT: Free and Cued Selective Reminding Test; GDS: Geriatric Depression Scale; HADS: Hospital Anxiety and Depression Scale; IADL: Instrumental activities of daily living; IDIBAPS: L'Institut d'Investigacions Biomèdiques August Pi i Sunyer Barcelona; M@T: Memory Alteration Test; MCl: Mild cognitive impairment; MMSE: Mini-Mental State Examination; MRI: Magnetic resonance imaging; NIA-AA: National Institute on Aging and the Alzheimer's Association; OR: Odds ratio; ptau181: Tau phosphorylated at position 181; RVLT: Rey Verbal Learning Test; SCD: Subjective cognitive decline; SD: Standard deviation; TMT: Trail-Making Test; t-tau: Total tau; VuMC: VU University Medical Center in Amsterdam

\section{Acknowledgements}

The authors would like to thank Manuela Thelen for her continued effort for the Euro-SCD project.

\section{Funding}

This work was supported by an EU Joint Program Neurodegenerative Disease Research (JPND) project (grant number JPND_PS_FP-689-019). The project is supported through the following funding organizations under the aegis of JPND (www.jpnd.eu): Germany, Bundesministerium für Bildung und Forschung (BMBF grant number 01ED1508); The Netherlands Organisation for Health Research and Development; Spain, Instituto De Salud Carlos III. This research was also supported by the German Research Council (DFG; grant number JE 2707/7-1) and the German Center for Neurodegenerative Diseases (Deutsches Zentrum für Neurodegenerative Erkrankungen; DZNE; reference number BN012) (DELCODE). WMvdF is the recipient of a research grant on subjective cognitive decline (SCIENCe project: Gieskes Strijbis fonds). Prof Jens Wiltfang is supported by an llídio Pinho professorship and iBiMED (UID/BIM 04501/2013), and FCT project PTDC/DTP_PIC/5587/2014 at the University of Aveiro, Portugal.

\section{Availability of data and materials}

The data which support this study are not publicly available, but may be provided upon reasonable request.

\section{Authors' contributions}

Conceptualization and design of the study: SW, JLM, MW, CET, LR, NCP, FHB, RERS, SAMS, LMPW, AD, WMvdF, and FJ; statistical analysis: SW, LMPW, NCP, and LR; interpretation of data: SW, JLM, MW, CET, LR, NCP, FHB, RERS, SAMS, $L M P W, A D, W M v d F$, and FJ; drafting and/or revision of manuscript: SW, JLM, MW, CET, LR, NCP, FHB, RERS, SAMS, LMPW, OP, KL, KB, JP, CL, ST, AS, MTH, $E D, A D, J W, W M v d F$, and FJ. All authors read and approved the final manuscript.

\section{Ethics approval and consent to participate}

The study protocol was approved by the ethical committees of all participating centers of the Euro-SCD project. For the IDIBAPS sample, this was the ethics committee of the Alzheimer's Disease and Other Cognitive Disorders Unit, Hospital Clinic of the University of Barcelona. Likewise, the VUmc local Medical Ethics Committee has approved a general protocol for biobanking and using the clinical data for research purposes. For the DELCODE multicenter study, approval was given by the ethical committees of the medical faculties of all participating sites: Berlin (Charité, University Medicine), Bonn, Cologne, Göttingen, Magdeburg, Munich (Ludwig-Maximilians-University), Rostock, and Tübingen. The process was led and coordinated by the ethical committee of the medical faculty of the University of Bonn. All patients provided written informed consent.

\section{Consent for publication}

Not applicable.

\section{Competing interests}

The authors declare that they have no competing interests.

\section{Publisher's Note}

Springer Nature remains neutral with regard to jurisdictional claims in published maps and institutional affiliations. 


\section{Author details}

'Department of Neurodegeneration and Geriatric Psychiatry, University Hospital Bonn, Bonn, Germany. ${ }^{2}$ German Center for Neurodegenerative Diseases, Sigmund-Freud-Straße 27, 53127 Bonn, Germany. ${ }^{3}$ Alzheimer's Disease and Other Cognitive Disorders Unit, IDIBAPS, Hospital Clinic, Barcelona, Spain. ${ }^{4}$ BarcelonaBeta Brain Research Center, Fundació Pasqual Maragall, Pompeu Fabra University, Barcelona, Spain. ${ }^{5}$ Neurochemistry Lab and Biobank, Department of Clinical Chemistry, Amsterdam Neuroscience, Amsterdam University Medical Centers, Amsterdam, The Netherlands. ${ }^{6}$ Alzheimer Center and Department of Neurology, Neuroscience Campus Amsterdam, Amsterdam University Medical Centers, Amsterdam, The Netherlands. 'Department of Psychiatry and Psychotherapy, Charité, Campus Benjamin Franklin, Berlin, Germany. ${ }^{8}$ Institute for Stroke and Dementia Research, Klinikum der Universität München, Ludwig-Maximilians-Universität, Munich, Germany. ${ }^{9}$ German Center for Neurodegenerative Diseases, Munich, Germany. ${ }^{10}$ Department of Neuropsychiatry, Charité-Universitaetsmedizin Berlin, Berlin, Germany. "Department of Psychiatry and Psychotherapy, University of Tübingen, Tübingen, Germany. ${ }^{12}$ German Center for Neurodegenerative Diseases, Tübingen, Germany. ${ }^{13}$ German Center for Neurodegenerative Diseases, Rostock, Germany. ${ }^{14}$ German Center for Neurodegenerative Diseases, Magdeburg, Germany. ${ }^{15}$ Department of Nuclear Medicine, Medical Faculty, University of Cologne, Cologne, Germany. ${ }^{16}$ German Center for Neurodegenerative Diseases (DZNE), Goettingen, Germany. ${ }^{17}$ Department of Psychiatry and Psychotherapy, University Medical Center Goettingen, Goettingen, Germany. ${ }^{18}$ Department of Epidemiology and Biostatistics, Amsterdam University Medical Centers, Amsterdam, The Netherlands. ${ }^{19}$ Massachusetts General Hospital, Department of Neurology / Harvard Medical School, Boston, USA. ${ }^{20}$ Department of Psychiatry, Medical Faculty, University of Cologne, Cologne, Germany. ${ }^{21}$ BBiMED, Medical Sciences Department, University of Aveiro, Aveiro, Portugal.

Received: 14 August 2018 Accepted: 20 December 2018 Published online: 17 January 2019

\section{References}

1. Andrieu S, Coley N, Lovestone S, Aisen PS, Vellas B. Prevention of sporadic Alzheimer's disease: lessons learned from clinical trials and future directions. Lancet Neurol. 2015;14:926-44. https://doi.org/10.1016/S14744422(15)00153-2.

2. Vellas B, Aisen PS, Sampaio C, Carrillo M, Scheltens P, Scherrer B, et al. Prevention trials in Alzheimer's disease: an EU-US task force report. Prog Neurobiol. 2011;95:594-600. https://doi.org/10.1016/j.pneurobio.2011.08.014.

3. Jack CR, Bennett DA, Blennow K, Carrillo MC, Dunn B, Haeberlein SB, et al. NIA-AA research framework: toward a biological definition of Alzheimer's disease. Alzheimers Dement. 2018;14:535-62. https://doi.org/10.1016/j.jalz. 2018.02.018.

4. Jessen F, Amariglio RE, van Boxtel M, Breteler M, Ceccaldi M, Chételat G, et al. A conceptual framework for research on subjective cognitive decline in preclinical Alzheimer's disease. Alzheimers Dement. 2014;10:844-52. https:// doi.org/10.1016/j.jalz.2014.01.001.

5. Molinuevo JL, Rabin LA, Amariglio R, Buckley R, Dubois B, Ellis KA, et al. Implementation of subjective cognitive decline criteria in research studies. Alzheimers Dement. 2017;13:296-311. https://doi.org/10.1016/j.jalz.2016.09.012.

6. van der Flier WM, Pijnenburg YAL, Prins N, Lemstra AW, Bouwman FH, Teunissen CE, et al. Optimizing patient care and research: the Amsterdam Dementia Cohort. J Alzheimers Dis. 2014;41:313-27. https://doi.org/10.3233/ JAD-132306.

7. van der Flier WM, Scheltens P. Amsterdam Dementia Cohort: performing research to optimize care. J Alzheimers Dis. 2018;62:1091-111. https://doi. org/10.3233/JAD-170850.

8. Sierra-Rio A, Balasa M, Olives J, Antonell A, Iranzo A, Castellví M, et al. Cerebrospinal fluid biomarkers predict clinical evolution in patients with subjective cognitive decline and mild cognitive impairment. Neurodegener Dis. 2016;16:69-76. https://doi.org/10.1159/000439258.

9. Jessen F, Spottke A, Boecker H, Brosseron F, Buerger K, Catak C, et al. Design and first baseline data of the DZNE multicenter observational study on predementia Alzheimer's disease (DELCODE). Alzheimers Res Ther. 2018;10: 15. https://doi.org/10.1186/s13195-017-0314-2.

10. Thalmann B, Monsch AU, Schneitter M, Bernasconi F, Aebi C, CamachovaDavet $Z$, et al. The CERAD neuropsychological assessment battery (CERAD-
NAB) — a minimal data set as a common tool for German-speaking Europe. Neurobiol Aging. 2000;21:30.

11. Reitan RM. Validity of the Trail Making Test as an indicator of organic brain damage. Percept Mot Skills. 1958;8:271-6.

12. Berres M, Monsch AU, Bernasconi F, Thalmann B, Stähelin HB. Normal ranges of neuropsychological tests for the diagnosis of Alzheimer's disease. Stud Health Technol Inform. 2000;77:195-9.

13. Petersen RC, Smith GE, Waring SC, Ivnik RJ, Tangalos EG, Kokmen E. Mild cognitive impairment: clinical characterization and outcome. Arch Neurol. 1999:56:303-8.

14. Rami L, Molinuevo JL, Sanchez-Valle R, Bosch B, Villar A. Screening for amnestic mild cognitive impairment and early Alzheimer's disease with M@T (Memory Alteration Test) in the primary care population. Int J Geriatr Psychiatry. 2007;22:294-304. https://doi.org/10.1002/gps.1672.

15. del Campo M, Mollenhauer B, Bertolotto A, Engelborghs S, Hampel H, Simonsen $\mathrm{AH}$, et al. Recommendations to standardize preanalytical confounding factors in Alzheimer's and Parkinson's disease cerebrospinal fluid biomarkers: an update. Biomark Med. 2012;6:419-30. https://doi.org/10. 2217/bmm.12.46.

16. Bertens D, Tijms BM, Scheltens P, Teunissen CE, Visser PJ. Unbiased estimates of cerebrospinal fluid $\beta$-amyloid 1-42 cutoffs in a large memory clinic population. Alzheimers Res Ther. 2017;9:8. https://doi.org/10.1186/ s13195-016-0233-7.

17. Tijms BM, Willemse EAJ, Zwan MD, Mulder SD, Visser PJ, Berckel v, Bart NM, et al. Unbiased approach to counteract upward drift in cerebrospinal fluid amyloid- $\beta$ 1-42 analysis results. Clin Chem. 2017. https://doi.org/10.1373/ clinchem.2017.281055.

18. Duits FH, Teunissen CE, Bouwman FH, Visser P, Mattsson N, Zetterberg H, et al. The cerebrospinal fluid "Alzheimer profile": easily said, but what does it mean? Alzheimers Dement. 2014;10:713-23.e2. https://doi.org/10.1016/j.jalz. 2013.12.023.

19. Rami L, Fortea J, Bosch B, Solé-Padullés C, Lladó A, Iranzo A, et al. Cerebrospinal fluid biomarkers and memory present distinct associations along the continuum from healthy subjects to AD patients. J Alzheimers Dis. 2011;23:319-26. https://doi.org/10.3233/JAD-2010-101422.

20. Saan RJ, Deelman BG. De 15-woordentest A en B (een voorlopige handleiding). Groningen: Afdeling Neuropsychologie, AZG; 1986.

21. Peña-Casanova J, Gramunt-Fombuena N, Quiñones-Ubeda S, SánchezBenavides G, Aguilar M, Badenes D, et al. Spanish Multicenter Normative Studies (NEURONORMA Project): norms for the Rey-Osterrieth complex figure (copy and memory), and free and cued selective reminding test. Arch Clin Neuropsychol. 2009;24:371-93. https://doi.org/10.1093/arclin/acp041.

22. Strauss E, Sherman EMS, Spreen O. A compendium of neuropsychological tests: administration, norms, and commentary. Oxford University Press: American Chemical Society; 2006.

23. Edmonds EC, Delano-Wood L, Galasko DR, Salmon DP, Bondi MW. Subtle cognitive decline and biomarker staging in preclinical Alzheimer's disease. J Alzheimers Dis. 2015;47:231-42. https://doi.org/10. 3233/JAD-150128.

24. Wagner M, Wolf S, Reischies FM, Daerr M, Wolfsgruber S, Jessen F, et al. Biomarker validation of a cued recall memory deficit in prodromal Alzheimer disease. Neurology. 2012;78:379-86. https://doi.org/10.1212/WNL. ob013e318245f447.

25. Yesavage JA, Sheikh JI. Geriatric depression scale (GDS): recent evidence and development of a shorter version. Clin Gerontol. 1986; 5:165-73.

26. Bjelland I, Dahl AA, Haug T, Neckelmann D. The validity of the Hospital Anxiety and Depression Scale. An updated literature review. J Psychosom Res. 2002:52:69-77.

27. Pfeffer Rl, Kurosaki TT, Harrah CH, Chance JM, Filos S. Measurement of functional activities in older adults in the community. J Gerontol. 1982;37:323-9.

28. Gélinas I, Gauthier L, Mclntyre M, Gauthier S. Development of a functional measure for persons with Alzheimer's disease: the disability assessment for dementia. Am J Occup Ther. 1999;53:471-81.

29. Mielke MM, Wiste HJ, Weigand SD, Knopman DS, Lowe VJ, Roberts RO, et al. Indicators of amyloid burden in a population-based study of cognitively normal elderly. Neurology. 2012;79:1570-7. https://doi.org/10.1212/WNL. 0b013e31826e2696.

30. Jessen F, Wolfsgruber S, Wiese B, Bickel H, Mösch E, Kaduszkiewicz H, et al. $\mathrm{AD}$ dementia risk in late $\mathrm{MCl}$, in early $\mathrm{MCl}$, and in subjective memory impairment. Alzheimers Dement. 2014;10:76-83. 
31. van Harten AC, Smits LL, Teunissen CE, Visser PJ, Koene T, Blankenstein MA, et al. Preclinical AD predicts decline in memory and executive functions in subjective complaints. Neurology. 2013;81:1409-16.

32. Willemse EAJ, van Uffelen KWJ, van der Flier WM, Teunissen CE. Effect of long-term storage in biobanks on cerebrospinal fluid biomarker Aß1-42, Ttau, and P-tau values. Alzheimers Dement (Amst). 2017;8:45-50. https://doi. org/10.1016/j.dadm.2017.03.005

33. Jutten RJ, Peeters CFW, Leijdesdorff SMJ, Visser PJ, Maier AB, Terwee CB, et al. Detecting functional decline from normal aging to dementia: development and validation of a short version of the Amsterdam IADL Questionnaire. Alzheimers Dement (Amst). 2017;8:26-35.

34. Papp KV, Amariglio RE, Mormino EC, Hedden T, Dekhytar M, Johnson KA, et al. Free and cued memory in relation to biomarker-defined abnormalities in clinically normal older adults and those at risk for Alzheimer's disease. Neuropsychologia. 2015;73:169-75. https://doi.org/10.1016/j. neuropsychologia.2015.04.034.

35. Papp KV, Mormino EC, Amariglio RE, Munro C, Dagley A, Schultz AP, et al. Biomarker validation of a decline in semantic processing in preclinical Alzheimer's disease. Neuropsychology. 2016;30:624-30. https://doi.org/10. 1037/neu0000246

Ready to submit your research? Choose BMC and benefit from:

- fast, convenient online submission

- thorough peer review by experienced researchers in your field

- rapid publication on acceptance

- support for research data, including large and complex data types

- gold Open Access which fosters wider collaboration and increased citations

- maximum visibility for your research: over $100 \mathrm{M}$ website views per year

At $\mathrm{BMC}$, research is always in progress.

Learn more biomedcentral.com/submissions 